\title{
Temporal Lobe Atrophy and White Matter Lesions are Related to Major Depression over 5 years in the Elderly
}

\author{
Pernille J Olesen*,', Deborah R Gustafson', Michela Simoni', Leonardo Pantoni ${ }^{2}$, Svante Östling', \\ Xinxin Guo' and Ingmar Skoog' \\ 'Neuropsychiatric Epidemiology Unit, Section for Psychiatry and Neurochemistry, Institute of Neuroscience and Physiology, Sahlgrenska Academy, \\ University of Gothenburg, Mölndal, Sweden; ${ }^{2}$ Department of Neurological and Psychiatric Sciences, University of Florence, Florence, Italy
}

\begin{abstract}
The influence of organic brain changes on the development of depression in the elderly is uncertain. Cross-sectional studies, most often from clinical samples, report associations with brain atrophy and cerebrovascular disease, while longitudinal population studies have given mixed results. Our aim was to investigate whether cortical atrophy and white matter lesions (WMLs) on computed tomography (CT) predict occurrence of depression in the elderly. This is a prospective population-based study with 5-year follow-up. The baseline sample included 525 elderly subjects, aged 70-86 years, without dementia or major depression, with a score on the Mini-Mental State Examination above 25, and without dementia at follow-up. Cortical atrophy and WMLs were evaluated at baseline using CT. The main outcome measure was development of major or minor depression at follow-up according to Diagnostic and Statistical Manual of Mental Disorders, fourth edition, as evaluated using neuropsychiatric examinations and hospital discharge registers. Logistic regression was used to estimate risk. Over the period of 5 years, 20 individuals developed major and 63 minor depression. Presence of temporal lobe atrophy (odds ratio $(O R)=2.81,95 \%$ confidence interval $(\mathrm{Cl})$ 1.04-7.62) and moderate-to-severe WMLs $(\mathrm{OR}=3.21,95 \% \mathrm{Cl} 1.00-10.26)$ independently predicted major, but not minor, depression after controlling for various confounders. Other brain changes did not predict occurrence of depression. Our findings suggest that temporal lobe atrophy and WMLs represent relatively independent and complementary pathways to major depression in the elderly. This may have implications for prevention, as both neurodegeneration and cerebrovascular disease have been related to preventable factors.
\end{abstract}

Neuropsychopharmacology (2010) 35, 2638-2645; doi:I0.1038/npp.2010.176; published online 29 September 2010

Keywords: depression; elderly; brain atrophy; white matter lesions; population; longitudinal

\section{INTRODUCTION}

Depression is one of the most common mental disorders in the elderly and has a significant impact on quality of life, mortality, and cognitive function (Palsson and Skoog, 1997). It is therefore important to learn more about its risk factors. Reported risk factors include female gender, adverse life events, other psychosocial factors, and somatic disorders, such as stroke and cardiovascular disorders (Djernes, 2006; Palsson and Skoog, 1997). In addition, it has been suggested that organic brain changes, such as neurodegeneration and cerebrovascular disease, may be important in the etiology of geriatric depression.

*Correspondence: Dr PJ Olesen, Neuropsychiatric Epidemiology Unit, Section for Psychiatry and Neurochemistry, Institute of Neuroscience and Physiology, Sahlgrenska Academy, University of Gothenburg, Wallinsgatan 6, 43141 Mölndal, Sweden, Tel: + 4631 3438642, Fax: + 4631 7760403, E-mail: pernille.olesen@neuro.gu.se

Received 27 May 2010; revised 6 August 2010; accepted 26 August 2010
Most studies on the association between brain atrophy and depression in the elderly have been cross-sectional and performed in clinical samples. The most consistent findings from cross-sectional studies are that depression in the elderly is associated with atrophy in the frontal and temporal lobes, especially hippocampal atrophy and ventricular enlargement (Schweitzer et al, 2001). Only one longitudinal population-based study examined the association between brain atrophy and occurrence of depressive symptoms or syndromes during follow-up (Ikram et al, 2010). This study reported a cross-sectional association between temporal and parietal lobe atrophy at baseline and concurrent depressive symptoms, and reported no association with depression occurring during follow-up. Another longitudinal study in elderly without dementia examined the association between mean depressive symptom scores over time and change in brain atrophy on magnetic resonance imaging (MRI) over a period of 9 years (Dotson et al, 2009). Cross-sectional associations between temporal and frontal lobe atrophy and depressive symptoms, and a longitudinal association between volume decline in frontal 
white matter and depressive symptom score over time were reported (Dotson et al, 2009). This study did not examine baseline brain atrophy in relation to incidence of depressive symptoms during follow-up.

Regarding cerebrovascular disease, cross-sectional studies, often from patient samples, consistently show associations between white matter lesions (WMLs) and depression (Greenwald et al, 1998; Ikram et al, 2010; Kumar et al, 2000; Kumar et al, 2002). The results from longitudinal population-based studies are conflicting. Three studies using MRI reported that WMLs increased the risk of subsequent depression (Godin et al, 2008; Steffens et al, 2002; Teodorczuk et al, 2007) while two could not confirm these findings (Ikram et al, 2010; Versluis et al, 2006).

It has been suggested that cortical atrophy and WMLs represent two relatively independent pathways to major depression in the elderly (Kumar et al, 2000; Kumar et al, 2002). This hypothesis was based on a cross-sectional clinical study, and has not been tested in longitudinal population-based studies. We examined brain atrophy and WMLs on computed tomography (CT) in a populationbased sample of elderly without depression or dementia, who were followed up for 5 years for the occurrence of major and minor depression.

\section{MATERIALS AND METHODS}

\section{Participants}

The study sample has been described in detail (Simoni et al, 2008), and was derived from the Prospective Population Study of Women (Bengtsson et al, 1973) and the Gerontological and Geriatric Population Studies (Skoog, 2004) in Gothenburg, Sweden. The participants were obtained from the Swedish Population Register on the basis of their birth date and were born in 1908, 1914, 1918, 1922, and 1930. Persons living in private households and institutions were included. In total, there were 1495 eligible individuals in 2000-2001, and 1051 accepted to participate (response rate $70.3 \%$ ). Of the 1051,1018 participated in a psychiatric examination and were invited for a CT scan. Of these, $642(63.1 \%)$ accepted to have a CT scan. The 642 individuals who participated in the CT examination were younger (mean age 74 vs 77 years, $p<0.001$ ), less often women $(71.3 \%$ vs $88.0 \%, p<0.001)$, and had a lower mortality rate from baseline to November $2003(3.1 \%$ vs $11.5 \%, p<0.001)$ compared with the 376 nonparticipants (Simoni et al, 2008). According to the Swedish Hospital Discharge Register, participants in CT examination experienced a lower frequency of dementia (0.8 vs $7.7 \%$, $p<0.001)$, any psychiatric diagnosis $(5.9$ vs $13.9 \%$, $p<0.001)$, and stroke $(5.6 \%$ vs $12.3 \%, p<0.001)$ compared with nonparticipants. There was no difference in the prevalence of major (3.0 vs $3.5 \%, p=0.66)$ or minor $(9.7$ vs $9.6 \%, p=0.97)$ depression between participants and nonparticipants.

A follow-up was performed in 2005-2006. Among those surviving, 459 accepted a new psychiatric examination (response rate $84.7 \%$ ). Those lost to follow-up (83 refusals and 21 who died) were traced for dementia, major and minor depression in the Swedish Hospital Discharge Register. Thus, the sample comprised 563 individuals.
Individuals with dementia $(n=16 ; 2.5 \%)$, major depression $(n=19 ; 3.0 \%)$, or a score on the Mini-Mental State Examination (MMSE) score $<26(n=44 ; 6.9 \%)$ at baseline were excluded. Those who developed dementia during follow-up $(n=38)$ were also excluded from further analyses, leaving 525 individuals.

Informed consent was obtained from all participants and/ or their relatives. The study was approved by the Ethics Committee for Medical Research at the University of Gothenburg.

\section{Neuropsychiatric Examinations and Interviews}

Both in 2000-2001 and in 2005-2006, trained psychiatric research nurses performed the semistructured neuropsychiatric examinations that included ratings of the past month's psychiatric signs and symptoms according to Comprehensive Psychopathological Rating Scale (Asberg et al, 1978), which is valid and reliable in elderly populations (van der Laan et al, 2005), and Mini-International Neuropsychiatric Interview (Sheehan et al, 1998), history of depression, and assessment of current medications. Ratings of common signs and symptoms of dementia were also carried out (eg, assessments of memory, orientation, general knowledge, apraxia, visuospatial function, understanding proverbs, following commands, naming ability, and language) and have been described in detail (Guo et al, 2007; Skoog et al, 1993). Cognitive function was also measured with the MMSE (Folstein et al, 1975). The psychiatric nurses who performed the examinations were supervised and trained by psychiatrists. In training sessions, nurses and psychiatrists rated symptoms and signs among both participants without dementia and those with dementia. Inter-rater reliability between psychiatrists and nurses was studied in 50 individuals. The $\kappa$-values for the presence $v s$ absence of signs and symptoms necessary to diagnose depression were between 0.62 and 1.00, indicating 'good' (reference range $\kappa=0.61-0.80$ ) or 'excellent' $(\kappa=0.81-1.00)$ agreement.

\section{Measurements of Covariates}

At baseline, participants underwent a physical examination and blood tests. Body mass index (BMI) was calculated as $\mathrm{kg} / \mathrm{m}^{2}$. Systolic and diastolic blood pressure (SBP and DBP) was measured in the right arm in the seated position after 5-min rest using a mercury manometer. DBP was defined as Korotkoff phase 5. Hypertension was defined as $\mathrm{SBP} \geqslant 140 \mathrm{~mm} \mathrm{Hg}$, and/or $\mathrm{DBP} \geqslant 90 \mathrm{~mm} \mathrm{Hg}$, and/or taking antihypertensive medication. Blood samples were taken after overnight fasting. Plasma glucose and serum cholesterol concentrations were measured. The diagnosis of stroke was based on information from self-reports, close informants, and the Swedish Hospital Discharge Register. Diagnoses of myocardial infarction, angina pectoris, and claudicatio intermittens were based on self-report and key informant interviews. Diabetes mellitus was defined based on a physician's diagnosis, being on antidiabetic therapy, or having two fasting venous or capillary whole blood glucose values $\geqslant 7.0 \mathrm{mmol} / \mathrm{l}$. 


\section{Diagnosis of Dementia and Depression}

Dementia was diagnosed by geriatric psychiatrists according to the Diagnostic and Statistical Manual of Mental Disorders (DSM), third edition, revised (American Psychiatric Association, 1987) on the basis of symptoms rated by the examiners during detailed neuropsychiatric examinations and close informant interviews, as described (Skoog et al, 1993). Diagnoses of major and minor depression according to DSM-IV (American Psychiatric Association, 1994) were determined from algorithms based on symptoms rated by the examiners during the psychiatric examinations. Depression was only diagnosed in the absence of dementia. Major depression excluded a diagnosis of minor depression. According to DSM-IV, major depression includes greater than or equal to five symptoms of depression during the same 2-week period, whereas minor depression includes two to four symptoms during the same 2 -week period. The symptom 'depressed mood' or 'loss of interest' is required for diagnoses.

The diagnosis of depression for individuals lost to followup was based on information from the Swedish Hospital Discharge Register. In Sweden, doctors register primary and secondary diagnoses according to the International Classification of Diseases, version 10 (ICD-10) (World Health Organization, 1993) on all patients discharged from hospitals. ICD-10 codes for major depression were F31. $4-5 / 32.2-3 / 33.2-3$ and for minor depression F31.3/32.0-1/ $32.8-9 / 33.0-1 / 33.8-9 / 34.1 / 34.8-9 / 38.0-1 / 41.2$.

\section{CT Scan Rating Methods}

The same CT scanner and scanning procedure were used for all scans. Continuous slices of $10 \mathrm{~mm}$ thickness were obtained. No contrast enhancement was used. The scans were evaluated by a neurologist (MS) experienced in CT and MRI ratings of WMLs and other cerebral lesions. The neurologist was blind to all clinical data.

The Gothenburg scale was used to rate WMLs (Skoog et al, 1994). According to this scale, WMLs are defined as periventricular or subcortical areas of decreased attenuation below that expected for normal white matter. The changes were always diffusely distributed within the white matter. The scale gives a global measure of WMLs in the brain, and has been used in our epidemiological studies since 1986 (Skoog et al, 1994). It is a 0- to 3-point scale that takes into account the severity of the hypodensity of WMLs, including 0 (absence of any attenuation), 1 (mild signal attenuation), 2 (moderate signal attenuation), and 3 (severe signal attenuation).

A subjective scale with three grades (absent, mild or moderate, and severe) was used to evaluate cortical atrophy. Location of atrophy was categorized as frontal, temporal, parietal, or occipital regions, according to the anatomical subdivision (Gustafson et al, 2004), and severity was scored according to the extent of sulcal widening (De Leon et al, 1980).

The intra-rater $\kappa$-values for the $\mathrm{CT}$ assessment were between 'fair' $(\kappa=0.21-0.40)$ and 'good' $(\kappa=0.61-0.80)$ for both WMLs and atrophy (Simoni et al, 2008). Interobserver agreement between the rater and a neuroradiologist was 'fair' $(\kappa=0.30)$ for WMLs, 'moderate' for temporal lobe atrophy $(\kappa=0.43)$, and 'fair' for the other cortical regions $(\kappa=0.29-0.36)$.

\section{Statistical Analysis}

The statistical analyses on the outcome major depression were based on participants without major depression or dementia at baseline, and who did not develop dementia during follow-up. In the analyses on minor depression, those with minor depression at baseline and those who developed major depression at follow-up were also excluded. WMLs were dichotomized as none, mild $v s$ moderate, or severe lesions. Cortical atrophy was dichotomized as absence $v s$ presence of atrophy in each brain region. The comparison group was free from both depression and dementia during follow-up. Sex and age categories (70 vs $>70$ years) were preselected covariates. Additional covariates that were evaluated included level of education $(0-7,8-10$, or $\geqslant 11$ years), baseline stroke, myocardial infarction, angina pectoris, claudicatio intermittens, hypertension, and diabetes mellitus, and levels of plasma glucose, serum cholesterol, BMI, SBP, and DBP.

The statistical analyses were performed with logistic regression models in two steps: first, we selected the covariates to be included in the final models by analyzing the association of each covariate with major depression, adjusting for gender. Factors were included in the final models if they were associated at the level of $p \leqslant 0.10$. This resulted in hypertension and serum cholesterol being included as covariates. Second, multivariate logistic regression analyses were performed estimating the odds of major or minor depression during follow-up in relation to cortical atrophy or WMLs at baseline and including age, sex, and the covariates selected in step one. Odds ratios (ORs) and 95\% confidence intervals (CI) were calculated.

We present the results for three models: Model I, without adjustments, Model II, adjusting for age, sex, and the covariates selected in step one; and Model III, including both cortical atrophy and WMLs in the same regression model. As a secondary analysis, we also present the results for Model III performed on a subsample of individuals with MMSE score $<25$ at both baseline and follow-up.

Statistical analyses were performed using SPSS version 16.0. Results were considered statistically significant at a level of $p<0.05$.

\section{RESULTS}

Baseline characteristics are given in Table 1. Of the 525 participants who were free from major depression and dementia at baseline, 20 developed major depression (19 were diagnosed at the follow-up examination and one from the hospital discharge register). In the analyses on minor depression, we also excluded 46 participants with minor depression at baseline as well as those who developed major depression, leaving 471 individuals. Among these, 63 developed minor depression (58 were diagnosed at the follow-up examination and 5 from the hospital discharge register). Results are presented for unadjusted (Table 2, Model I) and adjusted (Table 2, Model II and III) models. 
Table I Baseline Characteristics of The Study Population and Those who Developed Major or Minor Depression Over 5-Year Follow-Up

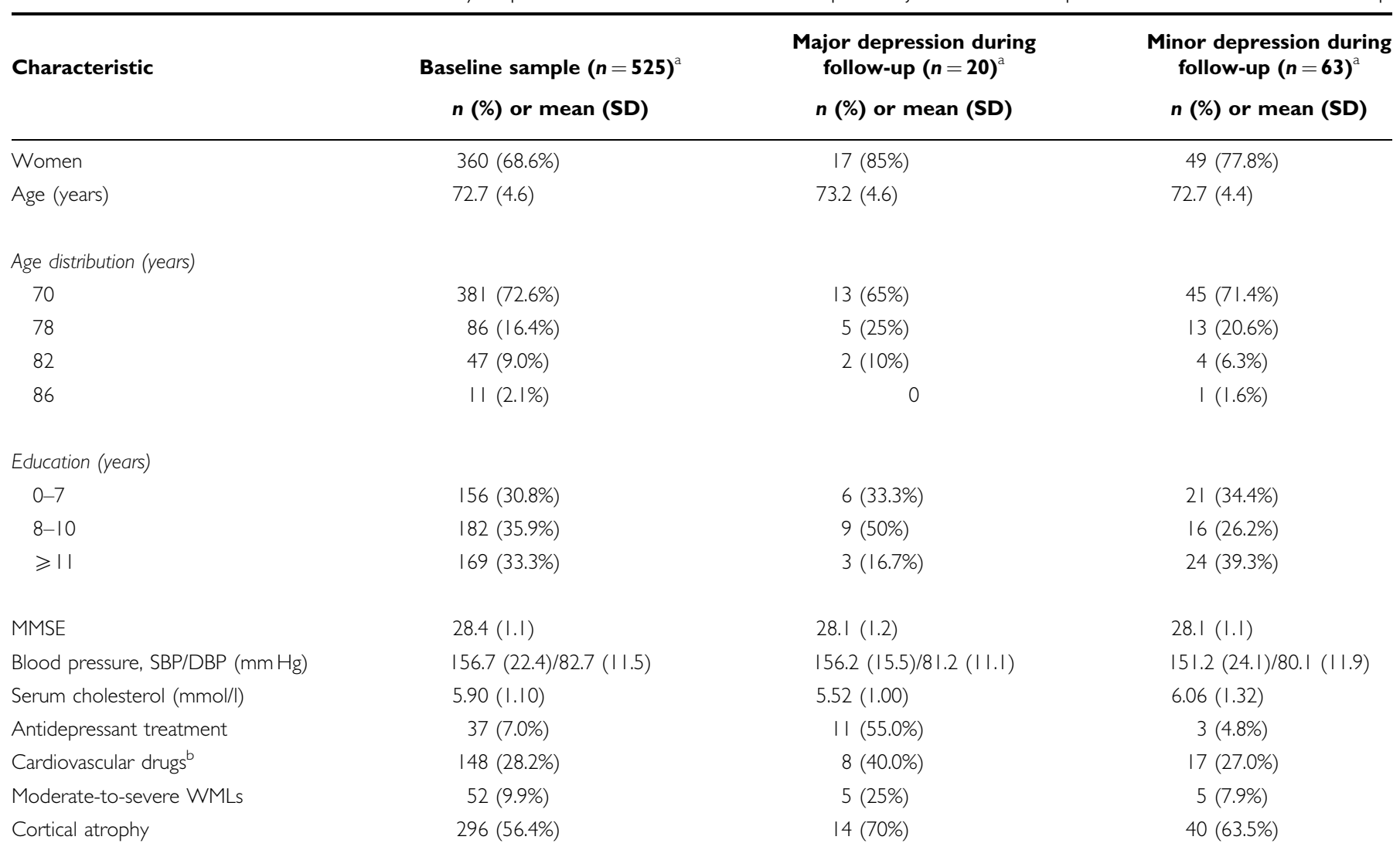

Abbreviations: DBP, diastolic blood pressure; MMSE, Mini-Mental State Examination; SBP, systolic blood pressure; WMLs, white matter lesions.

${ }^{a}$ Owing to missing values, the group sizes of the following measures were reduced: baseline sample-education $(n=507)$, blood pressure ( $\left.n=520\right)$, and serum cholesterol $(n=522)$; major depression-education $(n=18)$; minor depression-education $(n=61)$ and serum cholesterol $(n=62)$.

${ }^{\mathrm{b}}$ Antihypertensives, anticoagulants, cholesterol- and triglyceride-lowering, and antidiabetics.

Table 2 Cortical Atrophy and WMLs at Baseline in Relation to 5-year Occurrence of Major Depression ${ }^{\mathrm{a}}$

\begin{tabular}{|c|c|c|c|c|c|}
\hline & \multicolumn{2}{|c|}{$n / \mathbf{N}(\%)^{b}$} & \multicolumn{3}{|c|}{ OR $(95 \% \mathrm{Cl})$} \\
\hline & No atrophy & Atrophy & Model I (unadjusted) & Model II & Model III \\
\hline \multicolumn{6}{|l|}{ Atrophy in cortical areas } \\
\hline Parietal & 13/383 (3.4) & $7 / 142(4.9)$ & $1.47(0.58-3.78)$ & - & - \\
\hline \multirow[t]{2}{*}{ Occipital } & 16/440 (3.6) & $4 / 85(4.7)$ & $1.31(0.43-4.02)$ & - & - \\
\hline & No/mild WMLs & Moderate/severe WMLs & & & \\
\hline$\overline{\text { WMLs }}$ & $15 / 473(3.2)$ & $5 / 52(9.6)$ & $3.25(1.13-9.34)^{\mathrm{e}}$ & $3.21(1.02-10.10)^{\mathrm{e}}$ & $3.21(1.00-10.26)^{6}$ \\
\hline
\end{tabular}

Abbreviations: Cl, confidence interval; ORs, odds ratios; WMLs, white matter lesions.

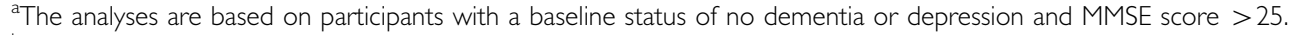

$b_{n / N}$ (within each group of cortical atrophy or WMLs) = (participants with major depression during follow-up)/(total number of participants).

'Adjusted for age, gender, baseline hypertension, and serum cholesterol. Results for Model II are presented only where Model I is significant.

${ }^{\mathrm{d}}$ Temporal lobe atrophy and WMLs in the same regression analysis. Adjusted for the same factors as in Model II.

$\mathrm{e}_{\mathrm{p}}<0.05$, Cox regression model.

Individuals with any temporal lobe atrophy at baseline more often developed major depression (6.4 vs 2.2\%, $\mathrm{OR}=2.81,95 \%$ CI 1.04-7.55) during follow-up than those with no atrophy (Table 2, Model II) adjusted for confounders (age, gender, baseline hypertension, and serum cholesterol). Similarly, individuals with moderate-to-severe 
WMLs more often developed major depression (9.6 vs 3.2\%, $\mathrm{OR}=3.21,95 \%$ CI 1.02-10.10) during follow-up (Table 2, Model II) compared with those with no or mild WMLs at baseline. There was no association between brain atrophy or WMLs and development of minor depression.

A logistic regression analysis, adjusted for confounders and including both temporal lobe atrophy and WMLs at baseline, showed that presence of temporal lobe atrophy $(\mathrm{OR}=2.81,95 \% \mathrm{CI}$ 1.04-7.62) and moderate-to-severe WMLs $(\mathrm{OR}=3.21,95 \%$ CI 1.00-10.26) independently predicted major depression at follow-up (Table 2, Model III). None of the confounders were significantly associated with major depression in this model. Excluding participants with MMSE score $<26$ at follow-up ( $n=35$; two with major depression) did not change the main results. Including baseline history of depression $(n=143)$ as confounder in the multivariate models did not change the findings.

\section{DISCUSSION}

We found that temporal lobe atrophy and moderate-tosevere WMLs predicted occurrence of major, but not minor, depression during a 5-year follow-up of a population-based sample of elderly. Our findings support the hypothesis that brain atrophy and WMLs represent relatively independent and complementary pathways to the development of major depression in the elderly (Kumar et al, 2000; Kumar et al, 2002). The only previous study that has explored this hypothesis is a clinical cross-sectional study, and depression was related to frontal but not temporal lobe atrophy (Kumar et al, 2000). Our study adds to this previous study by providing longitudinal data from a population-based sample. Both neurodegenerative and cerebrovascular processes may thus be important for the development of major depression in the elderly.

Only one previous longitudinal population-based study has examined the association between brain atrophy at baseline and depressive symptoms or syndromes during follow-up (Ikram et al, 2010). This study reported crosssectional associations between temporal and parietal lobe atrophy and depressive symptoms, but no association with depressive syndrome or depressive symptoms during follow-up. A longitudinal MRI study on elderly without dementia examined the association between mean depressive symptom scores over time and change in brain atrophy over 9 years (Dotson et al, 2009). In that study (Dotson et al, 2009), cross-sectional analyses indicated associations between temporal and frontal lobe atrophy and depressive symptoms, and longitudinal analyses indicated an association between volume decline in frontal white matter and mean depressive symptom scores over time. However, in contrast to our study, brain atrophy at baseline was not reported in relation to occurrence of depression or depressive symptoms during follow-up. The somewhat inconsistent findings between these previous longitudinal studies and our study may partly be explained by the fact that we report separately on major and minor depression rather than on depressive syndrome or depressive symptoms.

Presence of temporal lobe atrophy, but not atrophy in other brain areas, predicted major depression. Support for the importance of the temporal lobe in major depression is found in cross-sectional hospital-based MRI studies reporting that temporal lobe atrophy is associated with major depression in elderly patients (Dolan et al, 1986; Rabins et al, 1991; Rainer et al, 2006; Wurthmann et al, 1995). However, major depression in cross-sectional MRI studies has also been associated with other brain regions (Almeida et al, 2003; Dolan et al, 1986; Rabins et al, 1991; Rainer et al, 2006; Schweitzer et al, 2001; Wurthmann et al, 1995), including atrophy in the frontal and parietal lobes. Differences between these cross-sectional studies and our longitudinal study, which may explain the inconsistent findings, include the estimate of atrophy (ie, measures of sulcal widening, visual rating of cortical atrophy, estimates of CSF volume, and so on), the severity of atrophy (eg, any atrophy, severe atrophy), the choice of brain regions studied, the diagnostic criteria for depression, and the age of the samples.

It is well known that fronto-subcortical circuits are important for depression (Drevets et al, 2008; Ressler and Mayberg, 2007). These circuits include limbic structures such as the amygdala, which has substantial projections to the temporal cortex (Price and Drevets, 2010). Damage to fronto-subcortical circuits may be caused by subcortical lesions or atrophy in cortical structures. Thus, one explanation for the associations between WMLs and temporal lobe atrophy and major depression could be that these alterations in the brain have caused damage to frontosubcortical circuits.

The cellular mechanisms that underlie the association between brain atrophy and depression in the elderly remain largely unknown. Owing to the few postmortem studies performed, the results should be interpreted cautiously. Nevertheless, in relation to temporal lobe involvement, changes in myelination, axonal growth, and synaptic function have been reported in the temporal cortex of patients with major depression (Aston et al, 2005). Atrophy in the temporal lobe may also reflect hippocampal alterations. Postmortem studies have reported on cellular alterations such as neuronal death, neuronal shrinkage, decrease in dendritic and glial density, and cell death in the frontal cortex and hippocampus of those with major depression (Hercher et al, 2009; Khundakar and Thomas, 2009). Further support for a role of the temporal lobe in major depression is findings from a functional MRI study showing that the temporal lobes are involved in regulation of emotions (Beauregard et al, 1998). Hippocampal atrophy in depression (Pittenger and Duman, 2008) and during normal aging (Lupien et al, 1998) has been associated with increased cortisol levels, potentially reflective of physiological and environmental stress. One animal study indicated that psychosocial stress could be related to cell death in the temporal cortex (Lucassen et al, 2004).

WMLs predicted major depression in addition to the effect of temporal lobe atrophy. Cross-sectional associations between WMLs and depression have been shown repeatedly, especially for MRI and mainly from clinical studies (Greenwald et al, 1998; Ikram et al, 2010; Kumar et al, 2000; Kumar et al, 2002), although not all studies support this association (Rainer et al, 2006; Skoog et al, 1994). Our finding that WMLs preceded major depression is in agreement with three previous longitudinal studies using 
MRI (Godin et al, 2008; Steffens et al, 2002; Teodorczuk et al, 2007). However, two longitudinal population-based studies did not support this association (Ikram et al, 2010; Versluis et al, 2006). WMLs most likely reflect ischemic demyelination (Pantoni and Garcia, 1997). The association between depression and changes in the white matter has given rise to the 'vascular depression' hypothesis (Alexopoulos et al, 1997), which postulates that depression in the elderly may have a vascular origin. Our results support this view, especially regarding major depression.

Brain atrophy and WMLs did not predict occurrence of minor depression during follow-up, suggesting that this less severe form of depression may have a different etiology than major depression (Beekman et al, 1995). Our findings suggest that structural neuropathological changes in the temporal lobes and WMLs are related to more severe depression rather than to the milder forms. This is supported by cross-sectional studies suggesting that temporal lobe atrophy (Kumar et al, 1998; Shah et al, 1998) may be related to severity of depression rather than to depression per se. However, minor depression was related to frontal lobe atrophy in the study by Kumar et al (1998). Differences may be explained by differences between population-based and clinical studies, cross-sectional and longitudinal studies, and the use of somewhat different diagnostic criteria. We diagnosed minor depression based on DSM-IV criteria $(n=58)$ or ICD codes $(n=5)$, while Kumar et al (1998) diagnosed only according to DSM-IV, with the additional requirement that the symptoms should be present for at least 1 month. Thus, they may have looked at a more severe form of the disease compared with our study. Further, they included only those who had their fist onset of depression after the age of 60 years.

The strengths of the study include the population-based sample, the longitudinal design, and the comprehensive examinations performed by experienced psychiatric nurses. Furthermore, to minimize the risk that brain changes reflected preclinical dementia rather than depression, we excluded participants with a MMSE score $<26$, both at baseline and at follow-up. We will discuss some limitations and methodological issues. First, participants in the CT scans had a lower mortality rate, experienced stroke less often, dementia, and other mental disorders according to the Swedish Hospital Discharge Register, compared with nonparticipants (Simoni et al, 2008), and may thus be healthier than the general population. However, there were no differences in hospitalization with major or minor depression between participants and nonparticipants. Second, we cannot exclude the possibility that those who developed depression would have developed dementia if we had included a longer follow-up. Third, some of the subgroups were rather small and results should be interpreted cautiously. Future studies on larger samples are necessary to confirm our findings. Fourth, attrition is an issue that must be considered in follow-up studies. However, our attrition rate at follow-up was only $15.3 \%$. To further reduce the influence of attrition, we used the Swedish Hospital Discharge Register to obtain diagnoses of depression in those lost to follow-up. However, this probably still underestimates the true incidence, as most cases of depression are not hospitalized. Fifth, visual rating of cortical atrophy and WMLs on CT is a rather crude method. The inter-rater $\kappa$-values for agreement between the rater and a neuroradiologist was 'fair' $(\kappa=0.30)$ for WMLs, 'moderate' for temporal lobe atrophy $(\kappa=0.43)$, and 'fair' for the other cortical regions $(\kappa=0.29-0.36)$. If anything, the lack of perfect agreement most likely attenuates the observed relationship. CT is less sensitive than MRI in detecting WMLs and is more severely affected by bonehardening artifacts, which is particularly severe in the region of the medial temporal lobe (Frisoni, 2001). Nonetheless, visual rating of the temporal lobe has been found to be highly predictive for the discrimination of Alzheimer's disease $v s$ control patients (Wahlund et al, 2000). Further, CT is better in delineating clinically relevant WMLs (Lopez et al, 1995) and comparable with MRI in detecting brain atrophy (Frisoni, 2001), and may be more suitable than MRI for the elderly, as it is less sensitive to motion artefacts. CT is also the most used brain-imaging tool worldwide. Sixth, all scans were rated by a single person, which may increase the risk for systematic error.

The results from this longitudinal population-based study suggest that temporal lobe atrophy and WMLs represent independent pathways to major depression in the elderly, even if they may have shared risk factors. The finding that neurodegeneration and cerebrovascular disease are related to major depression may have implications for treatment and prevention, as these changes have been related to preventable factors, such as hypertension and obesity.

\section{ACKNOWLEDGEMENTS}

We thank Tomas Marlow and Kristoffer Bäckman for statistical assistance, both at the Neuropsychiatric Epidemiology Unit, University of Gothenburg, Sweden. This study was funded by grants from the Swedish Research Council (No. 11267, 2005-8460, 825-2007-7462), the Swedish Council for Working Life and Social Research (No. 2001-2835, 2001-2646, 2003-0234, 2004-0150, 20060020, 2006-1506, 2004-0145, 2006-0596, 2008-1111, 20081229), The Bank of Sweden Tercentenary Foundation, the Alzheimer's Association Zenith Award (ZEN-01-3151), the Alzheimer's Association Stephanie B. Overstreet Scholars (IIRG-00-2159), the Swedish Brain Power, Sylwans stiftelse, Söderström Königska, Fredrik och Ingrid Thurings stiftelse, Selma Anderssons stiftelse and the EU FP7 project LipiDiDiet (Grant agreement no. 211696). The funders had no role in the planning of the study or interpretation of the results.

\section{DISCLOSURE}

DRG has received honoraria from the Albuquerque Area Indian Health Board and Shire Pharmaceutical, and receives research support from the NIH (NIA 5R03AG026098 (PI)) and the Swedish Research Council. LP serves on the editorial boards of Acta Neurologica Scandinavica and International Journal of Alzheimer Disease. IS has served on scientific advisory boards for Pfizer and AstraZeneca; has served on an editorial advisory board for International Psychogeriatrics; receives royalties from publishing Alzheimers sjukdom och andra kognitiva sjukdomar (English title: Alzheimer's Disease and Other Cognitive Disorders (Liber 
2003)); serves on speakers' bureaus for Shire plc, JanssenCilag, Pfizer, Novartis, and Esai; and has received research support from the Swedish Research Council, the Alzheimer's Association, and the Bank of Sweden Tercentenary Foundation. The authors PJO, MS, SÖ, and XG report no biomedical financial interests or potential conflicts of interest.

\section{REFERENCES}

Alexopoulos GS, Meyers BS, Young RC, Campbell S, Silbersweig D, Charlson M (1997). 'Vascular depression'. hypothesis. Arch Gen Psychiatry 54: 915-922.

Almeida OP, Burton EJ, Ferrier N, McKeith IG, O’Brien JT (2003). Depression with late onset is associated with right frontal lobe atrophy. Psychol Med 33: 675-681.

American Psychiatric Association (1987). Diagnostic and Statistical Manual of Mental Disorders, 3rd edn, revised American Psychiatric Association: Washington, DC.

American Psychiatric Association (1994). Diagnostic and Statistical Manual of Mental Disorders, 4th edn American Psychiatric Press: Washington, DC.

Asberg M, Montgomery SA, Perris C, Schalling D, Sedvall G (1978). A comprehensive psychopathological rating scale. Acta Psychiatr Scand Suppl 57: 5-27.

Aston C, Jiang L, Sokolov BP (2005). Transcriptional profiling reveals evidence for signaling and oligodendroglial abnormalities in the temporal cortex from patients with major depressive disorder. Mol Psychiatry 10: 309-322.

Beauregard M, Leroux JM, Bergman S, Arzoumanian Y, Beaudoin G, Bourgouin P et al (1998). The functional neuroanatomy of major depression: an fMRI study using an emotional activation paradigm. Neuroreport 9: 3253-3258.

Beekman AT, Deeg DJ, van Tilburg T, Smit JH, Hooijer C, van Tilburg W (1995). Major and minor depression in later life: a study of prevalence and risk factors. J Affect Disord 36: 65-75.

Bengtsson C, Blohme G, Hallberg L, Hallstrom T, Isaksson B, Korsan-Bengtsen $\mathrm{K}$ et al (1973). The study of women in Gothenburg 1968-1969-a population study. General design, purpose and sampling results. Acta Med Scand 193: 311-318.

De Leon MJ, Ferris SH, George AE, Reisberg B, Kricheff II, Gershon $S$ (1980). Computed tomography evaluations of brain-behavior relationships in senile dementia of the Alzheimer's type. Neurobiol Aging 1: 69-79.

Djernes JK (2006). Prevalence and predictors of depression in populations of elderly: a review. Acta Psychiatr Scand 113: 372-387.

Dolan RJ, Calloway SP, Thacker PF, Mann AH (1986). The cerebral cortical appearance in depressed subjects. Psychol Med 16: 775-779.

Dotson VM, Davatzikos C, Kraut MA, Resnick SM (2009). Depressive symptoms and brain volumes in older adults: a longitudinal magnetic resonance imaging study. J Psychiatry Neurosci 34: 367-375.

Drevets WC, Price JL, Furey ML (2008). Brain structural and functional abnormalities in mood disorders: implications for neurocircuitry models of depression. Brain Struct Funct 213: 93-118.

Folstein MF, Folstein SE, McHugh PR (1975). 'Mini-mental state'. A practical method for grading the cognitive state of patients for the clinician. J Psychiatr Res 12: 189-198.

Frisoni GB (2001). Structural imaging in the clinical diagnosis of Alzheimer's disease: problems and tools. J Neurol Neurosurg Psychiatry 70: 711-718.

Godin O, Dufouil C, Maillard P, Delcroix N, Mazoyer B, Crivello F et al (2008). White matter lesions as a predictor of depression in the elderly: the 3C-Dijon study. Biol Psychiatry 63: 663-669.
Greenwald BS, Kramer-Ginsberg E, Krishnan KR, Ashtari M, Auerbach C, Patel M (1998). Neuroanatomic localization of magnetic resonance imaging signal hyperintensities in geriatric depression. Stroke 29: 613-617.

Guo X, Waern M, Sjogren K, Lissner L, Bengtsson C, Bjorkelund C et al (2007). Midlife respiratory function and Incidence of Alzheimer's disease: a 29-year longitudinal study in women. Neurobiol Aging 28: 343-350.

Gustafson D, Lissner L, Bengtsson C, Bjorkelund C, Skoog I (2004). A 24-year follow-up of body mass index and cerebral atrophy. Neurology 63: 1876-1881.

Hercher C, Turecki G, Mechawar N (2009). Through the looking glass: examining neuroanatomical evidence for cellular alterations in major depression. J Psychiatr Res 43: 947-961.

Ikram MA, Luijendijk HJ, Vernooij MW, Hofman A, Niessen WJ, van der Lugt A et al (2010). Vascular brain disease and depression in the elderly. Epidemiology 21: 78-81.

Khundakar AA, Thomas AJ (2009). Morphometric changes in early- and late-life major depressive disorder: evidence from postmortem studies. Int Psychogeriatr 21: 844-854.

Kumar A, Bilker W, Jin Z, Udupa J (2000). Atrophy and high intensity lesions: complementary neurobiological mechanisms in late-life major depression. Neuropsychopharmacology 22: 264-274.

Kumar A, Jin Z, Bilker W, Udupa J, Gottlieb G (1998). Late-onset minor and major depression: early evidence for common neuroanatomical substrates detected by using MRI. Proc Natl Acad Sci USA 95: 7654-7658.

Kumar A, Mintz J, Bilker W, Gottlieb G (2002). Autonomous neurobiological pathways to late-life major depressive disorder: clinical and pathophysiological implications. Neuropsychopharmacology 26: 229-236.

Lopez OL, Becker JT, Jungreis CA, Rezek D, Estol C, Boller F et al (1995). Computed tomography-but not magnetic resonance imaging-identified periventricular white-matter lesions predict symptomatic cerebrovascular disease in probable Alzheimer's disease. Arch Neurol 52: 659-664.

Lucassen PJ, Fuchs E, Czeh B (2004). Antidepressant treatment with tianeptine reduces apoptosis in the hippocampal dentate gyrus and temporal cortex. Biol Psychiatry 55: 789-796.

Lupien SJ, de Leon M, de Santi S, Convit A, Tarshish C, Nair NP et al (1998). Cortisol levels during human aging predict hippocampal atrophy and memory deficits. Nat Neurosci 1: 69-73.

Palsson S, Skoog I (1997). The epidemiology of affective disorders in the elderly: a review. Int Clin Psychopharmacol 12: S3-13.

Pantoni L, Garcia JH (1997). Pathogenesis of leukoaraiosis: a review. Stroke 28: 652-659.

Pittenger C, Duman RS (2008). Stress, depression, and neuroplasticity: a convergence of mechanisms. Neuropsychopharmacology 33: $88-109$.

Price JL, Drevets WC (2010). Neurocircuitry of mood disorders. Neuropsychopharmacology 35: 192-216.

Rabins PV, Pearlson GD, Aylward E, Kumar AJ, Dowell K (1991). Cortical magnetic resonance imaging changes in elderly inpatients with major depression. Am J Psychiatry 148: 617-620.

Rainer MK, Mucke HA, Zehetmayer S, Krampla W, Kuselbauer T, Weissgram S et al (2006). Data from the VITA Study do not support the concept of vascular depression. Am J Geriatr Psychiatry 14: 531-537.

Ressler KJ, Mayberg HS (2007). Targeting abnormal neural circuits in mood and anxiety disorders: from the laboratory to the clinic. Nat Neurosci 10: 1116-1124.

Schweitzer I, Tuckwell V, Ames D, O’Brien J (2001). Structural neuroimaging studies in late-life depression: a review. World $J$ Biol Psychiatry 2: 83-88. 
Shah PJ, Ebmeier KP, Glabus MF, Goodwin GM (1998). Cortical grey matter reductions associated with treatment-resistant chronic unipolar depression. Controlled magnetic resonance imaging study. Br J Psychiatry 172: 527-532.

Sheehan DV, Lecrubier Y, Sheehan KH, Amorim P, Janavs J, Weiller E et al (1998). The Mini-International Neuropsychiatric Interview (M.I.N.I.): the development and validation of a structured diagnostic psychiatric interview for DSM-IV and ICD-10. J Clin Psychiatry 59(Suppl 20): 22-33; quiz 34-57.

Simoni M, Pantoni L, Pracucci G, Palmertz B, Guo X, Gustafson D et al (2008). Prevalence of CT-detected cerebral abnormalities in an elderly Swedish population sample. Acta Neurol Scand 118: 260-267.

Skoog I (2004). Psychiatric epidemiology of old age: the H70 study-the NAPE lecture 2003. Acta Psychiatr Scand 109: 4-18.

Skoog I, Nilsson L, Palmertz B, Andreasson LA, Svanborg A (1993). A population-based study of dementia in 85-year-olds. $\mathrm{N} \mathrm{Engl} \mathrm{J}$ Med 328: 153-158.

Skoog I, Palmertz B, Andreasson LA (1994). The prevalence of white-matter lesions on computed tomography of the brain in demented and nondemented 85-year-olds. J Geriatr Psychiatry Neurol 7: 169-175.

Steffens DC, Krishnan KR, Crump C, Burke GL (2002). Cerebrovascular disease and evolution of depressive symptoms in the cardiovascular health study. Stroke 33: 1636-1644.
Teodorczuk A, O’Brien JT, Firbank MJ, Pantoni L, Poggesi A, Erkinjuntti $\mathrm{T}$ et al (2007). White matter changes and late-life depressive symptoms: longitudinal study. Br J Psychiatry 191: 212-217.

Wahlund LO, Julin P, Johansson SE, Scheltens P (2000). Visual rating and volumetry of the medial temporal lobe on magnetic resonance imaging in dementia: a comparative study. J Neurol Neurosurg Psychiatry 69: 630-635.

van der Laan NC, Schimmel A, Heeren TJ (2005). The applicability and the inter-rater reliability of the Comprehensive Psychopathological Rating Scale in an elderly clinical population. Int J Geriatr Psychiatry 20: 35-40.

Versluis CE, van der Mast RC, van Buchem MA, Bollen EL, Blauw GJ, Eekhof JA et al (2006). Progression of cerebral white matter lesions is not associated with development of depressive symptoms in elderly subjects at risk of cardiovascular disease: The PROSPER Study. Int J Geriatr Psychiatry 21: 375-381.

World Health Organization (1993). The ICD-10 Classification of Mental and Behavioural Disorders: Diagnostic Criteria for Research. World Health Organization: Geneva.

Wurthmann C, Bogerts B, Falkai P (1995). Brain morphology assessed by computed tomography in patients with geriatric depression, patients with degenerative dementia, and normal control subjects. Psychiatry Res 61: 103-111. 